\title{
RAIRO
}

ANALYSE NUMÉRIQUE

\section{IVAN HLAVÁČEK}

\section{A finite element solution for plasticity with strain-hardening}

RAIRO - Analyse numérique, tome 14, $\mathrm{n}^{\mathrm{O}} 4$ (1980), p. 347-368.

<http://www.numdam.org/item?id=M2AN_1980_14_4_347_0>

(C) AFCET, 1980, tous droits réservés.

L'accès aux archives de la revue «RAIRO - Analyse numérique » implique l'accord avec les conditions générales d'utilisation (http://www.numdam.org/ legal.php). Toute utilisation commerciale ou impression systématique est constitutive d'une infraction pénale. Toute copie ou impression de ce fichier doit contenir la présente mention de copyright.

\section{Numdam}

Article numérisé dans le cadre du programme

Numérisation de documents anciens mathématiques

http://www.numdam.org/ 


\title{
A FINITE ELEMENT SOLUTION FOR PLASTICITY WITH STRAIN-HARDENING ( $\left.{ }^{*}\right)$
}

\author{
by Ivan HLAVÁČEK $\left({ }^{1}\right)$
}

Communique par $\mathrm{P}$ G Ciarlet

\begin{abstract}
Three basic boundary value problems are formulated in terms of stresses and hardening parameters Plecewise linear simplicial elements are employed and some error estimates derived, provided certain regularty of the exact solution holds If the solution is not regular, the convergence is proven
\end{abstract}

Resume - Trols problemes aux limites fondamentau sont exprimc en fonc ton des contrainter et de parameties decroulssage On utilise des element, fins simplician lineaures pal monceau et on etablit des majorations d'erreur, moyennant une certaine regularite de la solution exacte Si la solution n'est pas regultere, on demontre la convergence

The flow theory of plasticity with strain-hardening material ( $c f$. [5]) has been studied recently by C. Johnson [8], Groger [3] and Nečas [6] from a new point of view, pioneered by Nguyen Quoc Son [14] and Halphen-Nguyen Quoc Son [4] The common idea of therr existence proofs is to formulate the problem by means of variational inequality of evolution and to use a penalty method.

Various incremental finite element solutions have been published in the engineering literature. To the author's knowledge, however, the only theoretical convergence analysis have been presented by C. Johnson [9] In the present paper, we propose another variant of the incremental finite element method, starting from the formulation of the quasi-static problem in terms of stresses and hardening parameters only. Whereas in the mixed method of [9] the stresses and hardening parameters are approximated by piecewise constant functions and the displacements by piecewise linear functions, we employ piecewise linear functions for both the stresses and the hardening parameters. The stress approximations consist of equilibriated triangular or tetrahedral blockelements respectively $(c f[15,7,11,12])$

$\left.{ }^{*}\right)$ Reçu novembre 1979

( $\left.{ }^{1}\right)$ Mathematical Institute of the Czechoslovak Academy of Sciences, Żitna 25, Praha 1, Tchecoslovaquie

R A I R O Analyse numerique/Numerical Analysıs, 0399-0516/1980/347/\$ 500

(C) Bordas-Dunod 
Following the approach of $\mathrm{C}$. Johnson $[9,10]$, we are able to prove some a priori error estimates for the three basic types of boundary conditions: the zero displacements on the whole boundary, the surface tractions on the whole boundary and the mixed case, provided certain regularity of the exact solution holds. Finally, we prove the convergence of the approximations without any regularity assumption.

\section{BASIC RELATIONS}

Let $\Omega$ be a polyhedral bounded domain in $R^{n}, n=2,3, x=\left(x_{1}, \ldots, x_{n}\right)$ a Cartesian coordinate system. Denote by $I=[0, T], 0<T<\infty$, a fixed interval of time. Let $\mathbb{R}_{\sigma}$ be the space of symmetric $n \times n$ matrices (stress tensors). A repeated index implies summation over the range $1, \ldots, n . C$ denotes a generic constant, which is not necessarily the same at different places.

Assume that a yield function $f: \mathbb{R}_{\sigma} \rightarrow R$ is given, which is convex, continuous in $\mathbb{R}_{\sigma}$, continuously differentiable in $\mathbb{R}_{\sigma} \dot{-}\{0\}$ and

$$
f(\lambda \sigma)=|\lambda| f(\sigma), \quad \forall \lambda \in R, \quad \forall \sigma \in \mathbb{R}_{\sigma} .
$$

Note that such function satisfies also the following condition

$$
\left|\partial f / \partial \sigma_{i j}\right|<C, \quad i, j=1, \ldots, n, \quad \forall \sigma \in \mathbb{R}_{\sigma} \dot{-}\{0\} .
$$

REMARK 1.1: An example of a function, satisfying all these conditions is the von Mises yield function

$$
f(\sigma)=\left(\sigma_{i j}^{\mathrm{D}} \sigma_{i j}^{\mathrm{D}}\right)^{1 / 2},
$$

where

$$
\sigma_{i j}^{\mathrm{D}}=\sigma_{i j}-\frac{1}{n} \delta_{i j} \sigma_{k k}
$$

is the stress deviatoric.

Let us introduce the following notations

$$
\begin{gathered}
\|\tau\|_{\mathbb{R}_{\sigma}}=\left(\tau_{i j} \tau_{i j}\right)^{1 / 2} \\
S=\left\{\tau: \Omega \rightarrow \mathbb{R}_{\sigma} \mid \tau_{i j} \in L^{2}(\Omega), \forall i, j\right\}, \\
\|\tau\|_{S}=\left(\int_{\Omega}\|\tau\|_{\mathbb{R}_{\sigma}}^{2} d x\right)^{1 / 2}, \quad H=S \times L^{2}(\Omega) .
\end{gathered}
$$

Let

$$
\partial \Omega=\bar{\Gamma}_{u} \cup \bar{\Gamma}_{\sigma}, \quad \Gamma_{u} \cap \Gamma_{\sigma}=\varnothing,
$$

where $\Gamma_{u}$ and $\Gamma_{\sigma}$ are either empty or open in $\partial \Omega$. 
Assume that a (reference) body force vector $F^{0} \in[C(\Omega)]^{n}$ and a (reference) surface traction vector $g^{0} \in\left[L^{2}\left(\Gamma_{\sigma}\right)\right]^{n}$ be given. If $\Gamma_{u}=\emptyset$, the total equilibrium conditions for $F^{0}, g^{0}$ are satisfied.

Let the actual body forces and surface tractions be

$$
\begin{aligned}
& F(t, x)=\gamma(t) F^{0}(x) \text { in } I \times \Omega, \\
& g(t, x)=\gamma(t) g^{0}(x) \quad \text { on } I \times \Gamma_{\sigma} .
\end{aligned}
$$

Here $\gamma: I \rightarrow R$ is a non-negative function from $C^{2}(I)$ such that

$$
\left.\begin{array}{ccc}
\exists t_{1}>0, \quad \gamma(t)=0, & \forall t \in\left[0, t_{1}\right], \\
\gamma(t) \in\left[\gamma\left(t_{n-1}\right), \gamma\left(t_{n}\right)\right] & \text { if } & \gamma\left(t_{n-1}\right) \leqq \gamma\left(t_{n}\right), \\
\gamma(t) \in\left[\gamma\left(t_{n}\right), \gamma\left(t_{n-1}\right)\right] & \text { if } & \gamma\left(t_{n}\right) \leqq \gamma\left(t_{n-1}\right),
\end{array}\right\}
$$

holds in any subinterval $I_{n}=\left[t_{n-1}, t_{n}\right]$ of all time discretizations, which will be considered in the following.

For any $t \in I$ we introduce the set of statically admissible stress tensors

$$
\begin{aligned}
E(t)=E(F(t), g(t))=\left\{\sigma \in S \mid \int_{\Omega} \sigma_{i j} e_{i j}(v) d x\right. & \\
& \left.=\int_{\Omega} F_{i}(t) v_{i} d x+\int_{\Gamma_{\sigma}} g_{i}(t) v_{i} d s, \forall v \in V\right\},
\end{aligned}
$$

where

$$
\begin{gathered}
V=\left\{v \in\left[H^{1}(\Omega)\right]^{n} \mid v=0 \text { on } \Gamma_{u}\right\} \\
e_{i j}(v)=\frac{1}{2}\left(\partial v_{i} / \partial x_{j}+\partial v_{j} / \partial x_{i}\right) .
\end{gathered}
$$

Let us define

$$
\begin{gathered}
\mathscr{F}(\tau, \alpha)=f(\tau)-\alpha, \\
B=\left\{(\tau, \alpha) \in \mathbb{R}_{\sigma} \times R \mid \mathscr{F}(\tau, \alpha) \leqq 0\right\}, \\
P=\{(\tau, \alpha) \in H \mid(\tau(x), \alpha(x)) \in B \text { a.e. in } \Omega\}, \\
K(t)=\left(E(t) \times L^{2}(\Omega)\right) \cap P, \quad t \in I .
\end{gathered}
$$

Let the elasticity coefficients $A_{i j k l} \in L^{\infty}(\Omega)$ be given $(i, j, k, l=1, \ldots, n)$ such that

$$
A_{i j k l}=A_{j i k l}=A_{k l i j}, \quad \text { a. e.in } \Omega
$$

and $\exists c_{0}>0$ such that

$$
A_{i j k l} \varepsilon_{i j} \varepsilon_{k l} \geqq c_{0} \varepsilon_{i j} \varepsilon_{i j}, \quad \forall \varepsilon \in \mathbb{R}_{\sigma}
$$

holds a. e. in $\Omega$.

vol. $14, n^{\circ} 4,1980$ 
Moreover, let positive constants $x \in R$ and $\alpha_{0} \in R$ be given.

We introduce the following bilinear forms for $\hat{\sigma}, \hat{\tau} \in H, \hat{\sigma}=(\sigma, \alpha), \hat{\tau}=(\tau, \beta)$ :

$$
\begin{gathered}
\langle\hat{\sigma}, \hat{\tau}\rangle=\sigma_{i j} \tau_{i j}+\alpha \beta, \quad|\hat{\tau}|=\langle\hat{\tau}, \hat{\tau}\rangle^{1 / 2}, \\
(\hat{\sigma}, \hat{\tau})_{0}=\int_{\Omega}\langle\hat{\sigma}, \hat{\tau}\rangle d x, \quad\|\hat{\sigma}\|=(\hat{\sigma}, \hat{\sigma})_{0}^{1 / 2}, \\
\{\hat{\sigma}, \hat{\tau}\}=\int_{\Omega} A_{i j k l} \sigma_{i j} \tau_{k l} d x+x \int_{\Omega} \alpha \beta d x, \\
\|\hat{\sigma}\|=\{\hat{\sigma}, \hat{\sigma}\}^{1 / 2} .
\end{gathered}
$$

Note that the norms $\|$.$\| and \|. \mid\|$ are equivalent. Denote by $\|\cdot\|_{0, \Omega}$ the norm in $L^{2}(\Omega)$.

Let $C_{0}^{1}(I, S)$ be the space of continuously differentiable mappings $\tau: I \rightarrow S$ such that $\tau(0)=0$. Let $H_{0}^{1}(I, S)$ be the closure of $C_{0}^{1}(I, S)$ by means of the norm

$$
\left(\int_{0}^{T}\left\|\frac{d \tau}{d t}\right\|_{S}^{2} d t\right)^{1 / 2}
$$

Similarly, let $H^{1}\left(I, L^{2}\right)$ be the closure of $C^{1}\left(I, L^{2}(\Omega)\right)$ by means of the norm

$$
\left(\int_{0}^{T}\left(\|\beta\|_{0, \Omega}^{2}+i d \beta / d t \|_{0, \Omega}^{2}\right) d t\right)^{1 / 2} .
$$

A weak solution of the plasticity problem with strain-hardening is a pair of functions

$$
\hat{\sigma} \equiv(\sigma, \alpha) \in H_{0}^{1}(I, S) \times H^{1}\left(I, L^{2}\right)
$$

such that

$$
\alpha(0)=\alpha_{0}, \quad \hat{\sigma}(t) \in K(t)
$$

and

$$
\{d \hat{\sigma}(t) / d t, \hat{\tau}-\hat{\sigma}(t)\} \geqq 0, \quad \forall \hat{\tau}=(\tau, \alpha) \in K(t)
$$

holds for a. e. $t \in I$.

The existence and uniqueness of a weak solution has been discussed in [6] for $\partial \Omega=\Gamma_{\sigma}$ and in [8] for $\partial \Omega=\Gamma_{u}$ (see also [3]). Let us recall, for instance, that in [6] the following condition

$$
\exists \tilde{\sigma} \in C_{0}^{1}(I, S), \quad \tilde{\sigma}(t) \in E(t), \quad \forall t \in I,
$$

is sufficient for the existence and uniqueness of a weak solution. 


\section{FINITE ELEMENT APPROXIMATIONS}

In the present section, some results of C. Johnson [10] will be extended to the case of plasticity with strain-hardening, using also several procedures of the same author, published in [8] and [9].

We shall use the following internal approximations of the set $E(t)$ :

$$
E_{h}(t)=\chi(t)+E_{h}^{0}, \quad 0<h \leqq h_{0}<\infty,
$$

where $\chi \in H_{0}^{1}(I, S)$ is a fixed stress field such that $\chi(t) \in E(t)$ a. e. in $I$ and $E_{h}^{0} \subset E(0,0)$ is a finite-dimensional subspace of self-equilibriated stress fields. Then $E_{h}(t) \subset E(t)$. For the existence of the functions $\chi$-see lemma 2.2.

Let $V_{h} \subset L^{2}(\Omega)$ be a finite-dimensional subspace, an approximation of $L^{2}(\Omega)$. Assume that $V_{h}$ contains constant functions.

Define

$$
K_{h}(t)=\left(E_{h}(t) \times V_{h}\right) \cap P
$$

so that $K_{h}(t) \subset K(t)$.

We introduce a discretization of the time interval as follows: let $N$ be a positive integer, $k=T / N, t_{n}=n k, n=0,1, \ldots, N, I_{n}=\left[t_{n-1}, t_{n}\right], \hat{\tau}^{n}=\hat{\tau}\left(t_{n}\right)$, $\partial \hat{\tau}^{n}=\left(\hat{\tau}^{n}-\hat{\tau}^{n-1}\right) / k$.

Instead of the problem (1.5) we introduce the following approximate problem: to find a $\hat{\sigma}_{h k}^{n} \in K_{h}\left(t_{n}\right)$ such that

$$
\left.\begin{array}{c}
\left\{\partial \hat{\sigma}_{h k}^{n}, \hat{\tau}-\hat{\sigma}_{h k}^{n}\right\} \geqq 0, \quad \forall \hat{\tau} \in K_{h}\left(t_{n}\right), \\
n=1, \ldots, N, \quad \hat{\sigma}_{h k}^{0}=\left(0, \alpha_{0}\right) .
\end{array}\right\}
$$

Since $\hat{\sigma}_{h k}^{n}$ minimizes the strictly convex functional

$$
\frac{1}{2}\|\hat{\sigma}\|^{2}-\left\{\hat{\sigma}, \hat{\sigma}^{n-1}\right\}
$$

over the closed convex set $K_{h}\left(t_{n}\right)$, there exists a unique $\hat{\sigma}_{h k}^{n}$, provided $K_{h}\left(t_{n}\right) \neq \emptyset$. From lemma 2.2 below we obtain a sufficient condition for $K_{h}(t) \neq \varnothing$, since $\xi(t) \in K_{h}(t)$.

First we prove an important lemma. Define

$$
\|q\|_{l^{2}(H)}=\left(\sum_{n=1}^{N}\left\|q^{n}\right\|^{2} k\right)^{1 / 2}
$$

for $q=\left(q^{1}, \ldots, q^{N}\right), q^{n} \in H$.

vol. $14, \mathrm{n}^{\circ} 4,1980$ 
Lemma 2. 1: Assume that, if $\Gamma_{\sigma} \neq \emptyset$, there exists a function

$$
\chi^{0} \in\left[L^{\infty}(\Omega)\right]^{n^{2}} \cap E\left(F^{0}, g^{0}\right) .
$$

Then positive constants $C, k_{0}$ exist such that

$$
\left\|\partial \hat{\sigma}_{h k}\right\|_{l^{2}(H)} \leqq C
$$

holds for any $k \leqq k_{0}$ and any $\left.\left.h \in\right] 0, h_{0}\right]$.

REMARK 2.1: Let $F^{0}$ be continuous in $\bar{\Omega}$. Then therê exists

$$
\chi^{1} \in S \cap\left[L^{\infty}(\Omega)\right]^{n^{2}}
$$

such that

$$
\operatorname{div} \chi^{1}=-F^{0} \text { in } \Omega
$$

( $\chi^{1}$ can be obtained by an integration).

Let the vector-function $g^{0}-\chi^{1} \cdot v$, where $v$ denotes the unit outward normal, be piecewise linear on $\Gamma_{\sigma}$ with respect to a simplicial partition of $\Gamma_{\sigma}$. Then the assumption (2.4) is satisfied.

In fact, there exists a simplicial partition of $\Omega$ and $\chi^{2} \in E_{h}^{0}$, where $E_{h}^{0}$ consists of piecewise linear stress fields $-c f$. section 3 below, such that

$$
\chi^{2} \cdot v=g^{0}-\chi^{1} \cdot v \text {. }
$$

Setting $\chi^{0}=\chi^{1}+\chi^{2}$, we obtain

$$
\begin{gathered}
\chi^{0} \in\left[L^{\infty}(\Omega)\right]^{n^{2}}, \quad \operatorname{div} \chi^{0}=-F^{0} \quad \text { in } \Omega, \\
\chi^{0} \cdot v=g^{0} \quad \text { on } \Gamma_{\sigma}
\end{gathered}
$$

which implies $\chi^{0} \in E\left(F^{0}, g^{0}\right)$.

The proof of lemma 2.1 is based on the following auxiliary lemmas.

LemMA 2. 2: Let (2.4) be satisfied. Then there exists

$$
\xi(t)=(\chi(t), \zeta(t)) \in K(t), \quad \forall t \in I, \quad \xi(0)=\left(0, \alpha_{0}\right)
$$

and positive constants $C, \delta_{1}$ such that

$$
\begin{gathered}
\sup _{t \in I}\left\|\frac{\partial^{j} \xi}{\partial t^{j}}\right\| \leqq C, \quad j=0,1,2 \\
\operatorname{dist}(\xi(x, t), \partial B) \geqq \delta_{1}, \quad \forall t \in I, \quad \text { a.e. in } \Omega .
\end{gathered}
$$

Proof: We use $\chi^{0}$ in case that $\Gamma_{\sigma} \neq \varnothing$ and

$$
\chi^{0} \in\left[L^{\infty}(\Omega)\right]^{n^{2}} \cap E\left(F^{0}\right)
$$


defined by integration of the equilibrium equation

$$
\operatorname{div} \chi^{0}=-F^{0}
$$

in case that $\Gamma_{\sigma}=\emptyset$.

Let us set

$$
\chi(t)=\gamma(t) \chi^{0}, \quad \zeta(t)=\gamma(t) C_{1}+\alpha_{0},
$$

where $C_{1}$ is a suitable constant.

Then obviously $\chi(t) \in E(t)$ holds in $I$ and

$$
f\left(\chi^{0}(x)\right) \leqq C_{1} \quad \text { a. e. in } \Omega
$$

holds for some $C_{1}>0$ by virtue of the boundedness of $\chi^{0}$. Consequently, we have

$$
\mathscr{F}(\chi, \zeta)=f(\chi)-\zeta=\gamma(t)\left[f\left(\chi^{0}\right)-C_{1}\right]-\alpha_{0} \leqq-\alpha_{0}<0
$$

for all $t \in I$ and almost all $x \in \Omega$. Hence

$$
\xi=(\chi, \zeta) \in P, \quad \forall t \in I, \quad \xi(t) \in K(t), \quad \xi(0)=\left(0, \alpha_{0}\right) .
$$

Since $\chi^{0}$ is bounded and $\gamma(t) \in C^{2}(I),(2.6)$ follows easily.

To verify (2.7), we realize that (2.8) and (1.2) imply that a fixed $\delta_{1}>0$ exists such that

$$
\mathscr{F}(\xi(t)+\rho) \leqq 0, \quad \forall \rho \in \mathbb{R}_{\sigma} \times R, \quad|\rho| \leqq \delta_{1} .
$$

Consequently, $\xi(t)+\rho \in B$ and (2.7) follows.

Q.E.D.

We shall prove lemma 2.1 by means of a penalty method. Let $\pi$ be the operator of the orthogonal projection in $\mathbb{R}_{\sigma} \times R$ onto the closed convex set $B$. Introduce the following penalty functional

$$
J_{\mu}(\hat{\tau})=\frac{1}{2 \mu}\|\hat{\tau}-\pi \hat{\tau}\|^{2}, \quad \mu>0, \quad \hat{\tau} \in H .
$$

Define new approximations

$$
\hat{\sigma}_{h k \mu}^{n} \in E_{h}\left(t_{n}\right) \times V_{h}, \quad n=0,1, \ldots, N
$$

by the equations (we omit the subscripts $h k \mu$ for the time being):

$$
\left.\begin{array}{c}
\left\{\partial \hat{\sigma}^{n}, \hat{\tau}\right\}+\left(J_{\mu}^{\prime}\left(\hat{\sigma}^{n}\right), \hat{\tau}\right)_{0}=0, \quad \forall \hat{\tau} \in E_{h}^{0} \times V_{h} . \\
\hat{\sigma}^{0}=\left(0, \alpha_{0}\right), \quad n=1, \ldots, N .
\end{array}\right\}
$$

Note that the Gâteaux derivative of $J_{\mu}$ is

$$
J_{\mu}^{\prime}(\hat{\sigma})=\frac{1}{\mu}(\hat{\sigma}-\pi \hat{\sigma})
$$

vol. $14, \mathrm{n}^{\circ} 4,1980$ 
The problem (2.10) has a unique solution for each $n$. In fact, $\hat{\sigma}^{n}$ minimizes the functional

$$
\frac{1}{2}\|\hat{\sigma}\| \|^{2}+k J_{\mu}(\hat{\sigma})-\left\{\hat{\sigma}^{n-1}, \hat{\sigma}\right\},
$$

which is coercive, convex and continuous, over the set $E_{h}\left(t_{n}\right) \times V_{h}$, which is closed and convex in $H$.

Lemma 2.3: Let (2.4) be satisfied. Then positive constants $C, k_{0}$ exist such that for $k \leqq k_{0}, 0<h \leqq h_{0}, \mu>0$ the following inequalities hold:

$$
\begin{gathered}
\max _{n=1, \ldots N}\left\|\hat{\sigma}_{h k \mu}^{n}\right\| \leqq C ; \\
\sum_{n=1}^{\mathrm{N}} k J_{\mu}\left(\hat{\sigma}_{h k \mu}^{n}\right) \leqq C ; \\
\sum_{n=1}^{\ddot{N} k} k\left\|J_{\mu}^{\prime}\left(\hat{\sigma}_{h k \mu}^{n}\right)\right\|_{L^{\mathrm{L}}(\Omega)} \leqq C,
\end{gathered}
$$

where

$$
\|f\|_{L^{1}(\Omega)}=\int_{\Omega}|f| d x \quad \text { for } f \in H .
$$

Proof: (i) Let us consider $\xi=(\chi, \zeta)$ from lemma 2.2 and set $\hat{\sigma}^{n}=\xi^{n}+\rho^{n}, n=0$, $1, \ldots, N$. Then we have

$$
\rho^{n}=\left(\bar{\sigma}^{n}, \beta^{n}\right), \quad \bar{\sigma}^{n} \in E_{h}^{0}, \quad \rho^{0}=(0,0) .
$$

Let us insert $\hat{\tau}=\rho^{n}$ in (2.10) and $\partial \hat{\sigma}^{n}=\partial \xi^{n}+\partial \rho^{n}$. We obtain

$$
\left\{\partial \rho^{n}, \rho^{n}\right\}+\left(J_{\mu}^{\prime}\left(\hat{\sigma}^{n}\right), \rho^{n}\right)_{0}=-\left\{\partial \xi^{n}, \rho^{n}\right\}, \quad n=1, \ldots, N .
$$

Since $J_{\mu}^{\prime}$ is monotone,

$$
\left(J_{\mu}^{\prime}\left(\hat{\sigma}^{n}\right), \rho^{n}\right)_{0}=\left(J_{\mu}^{\prime}\left(\hat{\sigma}^{n}\right)-J_{\mu}^{\prime}\left(\xi^{n}\right), \rho^{n}\right)_{0} \geqq 0 .
$$

Consequently, we may write

$$
\sum_{n=1}^{M} k\left\{\partial \rho^{n}, \rho^{n}\right\} \leqq-\sum_{n=1}^{M} k\left\{\partial \xi^{n}, \rho^{n}\right\}, \quad M=1, \ldots, N .
$$

On the other hand, we have

$$
\begin{aligned}
\sum_{n=1}^{M} k\left\{\partial \rho^{n}, \rho^{n}\right\}=\sum_{n=1}^{M} \frac{1}{2}\left(\left\|\left|\rho^{n}\left\|\left.\right|^{2}-\right\| \rho^{n-1} \|^{2}\right.\right.\right. & \\
& \left.+\left\|\rho^{n}-\rho^{n-1} \mid\right\|^{2}\right) \geqq\left.\frac{1}{2}\left\|\rho^{M}\right\|\right|^{2}
\end{aligned}
$$


and therefore, by virtue of (2.6), we may write

$$
\begin{aligned}
\sum_{n=1}^{M} k\left|\left\{\partial \xi^{n}, \rho^{n}\right\}\right| \leqq C k & \sum_{n=1}^{M}\left\|\rho^{n}\right\| \mid \\
& \leqq \frac{1}{2} C k \sum_{n=1}^{M}\left(1+\|\| \rho^{n} \|\left.\right|^{2}\right) \leqq C+C k \sum_{n=1}^{M}\|\| \rho^{n}|\||^{2}
\end{aligned}
$$

We employ the following discrete analogue of Gronwall's lemma (see [1], chapt. 3, lemma 3.3) :

let

Then

$$
\begin{gathered}
\varphi(M) \leqq \psi(M)+\sum_{m=0}^{M-1} \chi(m) \varphi(m), \quad M=1, \ldots, n \leqq N, \\
\chi(m) \geqq 0, \quad \forall m .
\end{gathered}
$$

$$
\varphi(n) \leqq \psi(n)+\sum_{m=0}^{n-1} \chi(m) \psi(m) \prod_{s=m+1}^{n-1}(1+\chi(s)) .
$$

From (2.13), (2.14) and (2.15) it follows

$$
\left\|\left|\rho^{M}\left\|\left.\right|^{2} \leqq C+C \sum_{m=1}^{M-1} k\right\| \rho^{m}\right|\right\|^{2}, \quad M=1, \ldots, N .
$$

Setting $\varphi^{m}=\left\|\rho^{m}\right\| \|^{2}, \psi(M)=C, \chi(m)=C k$, we obtain

$$
\left\|\left|\rho^{n} \|\right|^{2} \leqq C+\sum_{m=0}^{n-1} C k(1+C k)^{n-1-m} \leqq C_{1},\right.
$$

because $(1+C k)^{N} \leqq C_{2}$ for $k \leqq k_{0}$.

Finally,

$$
\left\|\hat{\sigma}^{n}\right\||\leqq|\left\|\rho^{n}\right\|\left|+\left\|\left|\xi^{n} \|\right| \leqq C, \quad n=1, \ldots, N\right.\right.
$$

follows from (2.6).

(ii) We use the convexity of $J_{\mu}$ and lemma 2.2 to obtain

$$
J_{\mu}\left(\hat{\sigma}^{n}\right)+\left(J_{\mu}^{\prime}\left(\hat{\sigma}^{n}\right), \xi^{n}-\hat{\sigma}^{n}\right)_{0} \leqq J_{\mu}\left(\xi^{n}\right)=0 .
$$

From (2.11), (2.12), (2.14) we deduce

$$
\sum_{n=1}^{N} k\left(J_{\mu}^{\prime}\left(\hat{\sigma}^{n}\right), \rho^{n}\right)_{0} \leqq-\sum_{n=1}^{N} k\left\{\partial \xi^{n}, \rho^{n}\right\} \leqq \sum_{n=1}^{N} k\left\|| \partial \xi ^ { n } | | | \cdot \left|\left\|\rho^{n}|\|| \leqq C T,\right.\right.\right.
$$

using also (2.16) and (2.6). Consequently,

$$
\sum_{n=1}^{N} k J_{\mu}\left(\hat{\sigma}^{n}\right) \leqq \sum_{n=1}^{N} k\left(J_{\mu}^{\prime}\left(\hat{\sigma}^{n}\right), \hat{\sigma}^{n}-\xi^{n}\right)_{0} \leqq C .
$$

vol. $14, n^{\circ} 4,1980$ 
(iii) Let us consider

$$
\hat{\sigma}^{n}(x)=\xi^{n}(x)+\rho^{n}(x) \notin B
$$

and define

$$
j(x)=\frac{\hat{\sigma}^{n}(x)-\pi \hat{\sigma}^{n}(x)}{\left|\hat{\sigma}^{n}(x)-\pi \hat{\sigma}^{n}(x)\right|} .
$$

$j(x)$ is the unit normal with respect to the hyperplane $L$, separating $B$ and $\hat{\sigma}^{n}$ at the point $\pi \hat{\sigma}(x)$, where

$$
L=\left\{\hat{\tau} \in \mathbb{R}_{\sigma} \times R \mid\langle j(x), \hat{\tau}\rangle=d\right\}
$$

and $d \in R$ is a constant.

Since $\hat{\sigma}^{n}(x) \notin B$ and $\xi^{n}(x)+\delta_{1} j(x) \in B$ follows from (2.7), we have

Hence

$$
\begin{aligned}
& \left\langle j(x), \xi^{n}(x)+\rho^{n}(x)\right\rangle \geqq d, \\
& \left\langle j(x), \xi^{n}(x)+\delta_{1} j(x)\right\rangle \leqq d .
\end{aligned}
$$

$$
\left\langle j, \rho^{n}\right\rangle \geqq d-\left\langle j, \xi^{n}\right\rangle \geqq \delta_{1}\langle j, j\rangle=\delta_{1} .
$$

Inserting the definition of $j$, we obtain

$$
\left|\hat{\sigma}^{n}(x)-\pi \hat{\sigma}^{n}(x)\right| \leqq \frac{1}{\delta_{1}}\left\langle\hat{\sigma}^{n}(x)-\pi \hat{\sigma}^{n}(x), \rho^{n}(x)\right\rangle .
$$

Obviousiy, the same inequality is irue even for $\hat{\sigma}^{n} \in B$.

Integration over $x \in \Omega$ yields that

$$
\begin{aligned}
\left\|J_{\mu}^{\prime}\left(\hat{\sigma}^{n}\right)\right\|_{L^{1}(\Omega)}=\frac{1}{\mu} \int_{\Omega}\left|\hat{\sigma}^{n}-\pi \hat{\sigma}^{n}\right| & d x \\
& \leqq \frac{1}{\delta_{1} \mu} \int_{\Omega}\left\langle\hat{\sigma}^{n}-\pi \hat{\sigma}^{n}, \rho^{n}\right\rangle d x=\frac{1}{\delta_{1}}\left(J_{\mu}^{\prime}\left(\hat{\sigma}^{n}\right), \rho^{n}\right)_{0} .
\end{aligned}
$$

The estimate (iii) is an easy consequence of (2.17).

Lemma 2.4: Let (2.4) be satisfied. Then positive constants $C, k_{0}$ and $h_{0}$ exist such that

$$
\left\|\partial \hat{\sigma}_{h k \mu}^{n}\right\|_{l^{2}(H)} \leqq C
$$

holds for any $k \leqq k_{0}, 0<h \leqq h_{0}, \mu>0$.

Proof: From (2.10) it follows that

$$
\left\{\partial^{2} \hat{\sigma}^{n}, \hat{\tau}\right\}+\left(\partial\left(J_{\mu}^{\prime}\left(\hat{\sigma}^{n}\right)\right), \hat{\tau}\right)_{0}=0, \quad \forall \hat{\tau} \in E_{h}^{0} \times V_{h},
$$

for $n=2, \ldots, N$. 
Choosing $\hat{\tau}=\partial \rho^{n}$ and inserting $\hat{\sigma}^{n}=\xi^{n}+\rho^{n}$, we obtain

$$
\left\{\partial^{2} \rho^{n}, \partial \rho^{n}\right\}+\left(\partial\left(J_{\mu}^{\prime}\left(\hat{\sigma}^{n}\right)\right), \partial \sigma^{n}\right)_{0}=-\left\{\partial^{2} \xi^{n}, \partial \rho^{n}\right\}+\left(\partial\left(J_{\mu}^{\prime}\left(\hat{\sigma}^{n}\right), \partial \xi^{n}\right)_{0} .\right.
$$

The second term on the left side is non-negative, due to the monotonicity of $J_{\mu}^{\prime}$. Summing by parts in the last term, we may write

$$
\begin{aligned}
\sum_{n=2}^{M} k\left\{\partial^{2} \rho^{n}, \partial \rho^{n}\right\} \leqq-\sum_{n=2}^{M} k\left\{\partial^{2} \xi^{n}, \partial \rho^{n}\right\} & \\
& -\sum_{n=2}^{M-1} k\left(J_{\mu}^{\prime}\left(\hat{\sigma}^{n}\right), \partial^{2} \xi^{n+1}\right)_{0}+\left(J_{\mu}^{\prime}\left(\hat{\sigma}^{M}\right), \partial \xi^{M}\right)_{0} .
\end{aligned}
$$

Here we have used the fact that $\partial \xi^{2}=\left(\xi^{2}-\xi^{1}\right) / k=0$ holds for $k \leqq(1 / 2) t_{1}$, by virtue of the definition of $\xi(t)$ and (1.3).

From (2.6) and lemma 2.3-(iii), it follows that

$$
\left|\sum_{n=2}^{M-1} k\left(J_{\mu}^{\prime}\left(\hat{\sigma}^{n}\right), \partial^{2} \xi^{n+1}\right)_{0}\right| \leqq C \sum_{n=2}^{M-1} k\left\|J^{\prime}\left(\hat{\sigma}^{n}\right)\right\|_{L^{1}(\Omega)} \leqq C_{1} .
$$

Furthermore,

$$
\sum_{n=2}^{M} k\left\{\partial^{2} \rho^{n}, \partial \rho^{n}\right\} \geqq \frac{1}{2}\left(\left\|\partial \rho^{M}\right\|^{2}-\left\|\mid \partial \rho^{1}\right\|^{2}\right),
$$

consequently, we have for any $M=2, \ldots, N$ :

$$
\left\|\left|\partial \rho^{M}\right|\right\|^{2} \leqq C+C\left(J_{\mu}^{\prime}\left(\hat{\sigma}^{M}\right), \partial \xi^{M}\right)_{0}+\left.\left\|\partial \rho^{1}\right\|\right|^{2}+\sum_{n=2}^{M} C k\|\| \rho^{n} \|\left.\right|^{2} .
$$

The discrete analogue of the Gronwall's lemma yields

$$
\left.\left\|\partial \partial \rho^{n}\right\|\right|^{2} \leqq C+C\left(J_{\mu}^{\prime}\left(\hat{\sigma}^{n}\right), \partial \xi^{n}\right)_{0}+C \sum_{m=1}^{n-1} k\left(J_{\mu}^{\prime}\left(\hat{\sigma}^{m}\right), \partial \xi^{m}\right)_{0},
$$

for $n=2, \ldots, N$. Using again lemma 2.3-(iii) in the last term, we obtain

$$
\begin{gathered}
\|\| \partial \rho^{n}\|\|^{2} \leqq C_{1}+C\left(J_{\mu}^{\prime}\left(\hat{\sigma}^{n}\right), \partial \xi^{n}\right)_{0}, \quad n=2, \ldots, N, \\
\sum_{n=2}^{N} k\left\|\partial \rho^{n} \mid\right\|^{2} \leqq C T+C \sum_{n=2}^{N} k\left(J_{\mu}^{\prime}\left(\hat{\sigma}^{n}\right), \partial \xi^{n}\right)_{0} \leqq C_{1} .
\end{gathered}
$$

Thus we may write

$$
\left.\begin{array}{c}
\left.\left\|\partial \hat{\sigma}^{n}\right\|\right|^{2} \leqq 2\|\| \partial \xi^{n}\left|\left\|^{2}+2\left|\left\|\partial \rho^{n}||^{2} \leqq C+2\left|\left\|\partial \rho^{n} \mid\right\|^{2},\right.\right.\right.\right.\right. \\
\left.\sum_{n=2}^{N} k\left\|\partial \hat{\sigma}^{n}\right\|\right|^{2} \leqq C T+2 \sum_{n=2}^{N} k\left\|\partial \rho^{n}\right\| \|^{2} \leqq C_{2} .
\end{array}\right\}
$$

It remains to prove that

$$
k\left|\left\|\partial \hat{\sigma}^{1} \mid\right\|^{2} \leqq C .\right.
$$

vol. $14, n^{\circ} 4,1980$ 
Inserting $\hat{\sigma}^{1}=\xi^{1}+\rho^{1}, \hat{\tau}=\partial \rho^{1}$ into (2.10), we obtain

$$
\|\| \partial \rho^{1}\|\|^{2}+\left\{\partial \xi^{1}, \partial \rho^{1}\right\}+\left(J_{\mu}^{\prime}\left(\hat{\sigma}^{1}\right), \partial \hat{\sigma}^{1}\right)_{0}-\left(J_{\mu}^{\prime}\left(\hat{\sigma}^{1}\right), \partial \xi^{1}\right)_{0}=0 .
$$

The third term is non-negative $\left[J_{\mu}^{\prime}\left(\hat{\sigma}^{0}\right)=0\right.$ since $\left.\hat{\sigma}^{0} \in B\right]$. Hence using (iii) of lemma 2.3, we deduce

$$
k\left\|\partial \rho^{1}\right\| \|^{2} \leqq C
$$

and (2.20) follows easily.

The estimate (2.18) is a consequence of (2.19), (2.20) and the equivalence of the norms $\||\cdot|\|$ and $\|$.$\| .$

Q E.D.

To prove lemma 2.1 , we now let $\mu$ tend to zero through some sequence of positive numbers. From lemma 2.3-(i) we obtain the existence of a positive constant $C$ such that

$$
\left\|\hat{\sigma}_{h k \mu}\right\|_{R^{2}(H)} \leqq C, \quad \forall \mu>0 .
$$

In fact, we have

$$
\left\|\hat{\sigma}_{h k \mu}\right\|_{l^{2}(H)} \leqq C N k \max _{n}\left\|\hat{\sigma}_{h k}^{n}\right\| \leqq C_{1} T .
$$

Consequently, there exists a subsequence of $\mu$ and $\hat{\sigma}_{h k}$ such that for $\mu \rightarrow 0$ :

$$
\hat{\sigma}_{h k \mu} \rightarrow \hat{\sigma}_{h k} \quad \text { (weakly) in } l^{2}(H) \text {. }
$$

From lemma 2.4 we conclude that for $\mu \rightarrow 0$ :

$$
\partial \hat{\sigma}_{h k \mu} \rightarrow \hat{s}_{h k} \quad \text { (weakly) in } l^{2}(H) .
$$

It is not difficult to verify that $\hat{S}_{h k}=\partial \hat{\sigma}_{h k}$.

We are going to prove that $\hat{\sigma}_{h k}$ is a solution of the problem (2.2). Since $J_{\mu}$ is convex, we have

$$
J_{\mu}\left(\hat{\tau}^{n}\right) \geqq J\left(\hat{\sigma}_{h k \mu}^{n}\right)+\left(J_{\mu}^{\prime}\left(\hat{\sigma}_{h k \mu}^{n}\right), \hat{\tau}^{n}-\hat{\sigma}_{h k \mu}^{n}\right)_{0} .
$$

If $\hat{\tau}^{n} \in E_{h}\left(t_{n}\right) \times V_{h}$, then $\hat{\tau}_{0} \equiv \hat{\tau}^{n}-\hat{\sigma}_{h k \mu}^{n} \in E_{h}^{0} \times V_{h}$ and we may use (2.10) to obtain (omitting the subscripts $h k \mu$ ):

$$
\left(J_{\mu}^{\prime}\left(\hat{\sigma}^{n}\right), \hat{\tau}^{n}-\hat{\sigma}^{n}\right)_{0}=-\left\{\partial \hat{\sigma}^{n}, \hat{\tau}^{n}-\hat{\sigma}^{n}\right\} .
$$

Consequently, we arrive at the inequality

$$
\left\{\partial \hat{\sigma}^{n}, \hat{\tau}^{n}-\hat{\sigma}^{n}\right\}+J_{\mu}\left(\hat{\tau}^{n}\right)-J_{\mu}\left(\hat{\sigma}^{n}\right) \geqq 0 .
$$

Setting $\hat{\tau}^{n} \in K_{h}\left(t_{n}\right)$, we obtain $J_{\mu}\left(\hat{\tau}^{n}\right)=0$ and

$$
\left\{\partial \hat{\sigma}^{n}, \hat{\tau}^{n}-\hat{\sigma}^{n}\right\} \geqq 0, \quad \forall \hat{\tau}^{n} \in K_{h}\left(t_{n}\right) .
$$

R.A.I.R.O. Analyse numérique/Numerical Analysis 
Hence we may write, using (2.21) and (2.22):

$$
\begin{gathered}
0 \leqq \limsup _{\mu \rightarrow 0}\left[-\sum_{n=1}^{M} k\left\{\partial \hat{\sigma}^{n}, \hat{\sigma}^{n}\right\}+\sum_{n=1}^{M} k\left\{\partial \hat{\sigma}^{n}, \hat{\tau}^{n}\right\}\right] \\
=\limsup _{\mu \rightarrow 0}\left[\frac{1}{2}\left\|\hat{\sigma}^{0}\right\|\left\|^{2}-\frac{1}{2}\right\| \hat{\sigma}^{M} \|^{2}\right. \\
\left.-\frac{1}{2} \sum_{n=1}^{M}\left\|\hat{\sigma}^{n}-\hat{\sigma}^{n-1}\right\|^{2}+\sum_{n=1}^{M} k\left\{\partial \hat{\sigma}^{n}, \hat{\tau}^{n}\right\}\right] \\
=\frac{1}{2}\left|\left\|\hat{\sigma}^{0}\right\|\left\|^{2}-\frac{1}{2}\right\| \hat{\sigma}_{h k}^{M}\left\|^{2}-\frac{1}{2} \sum_{n=1}^{M}\right\| \hat{\sigma}_{h k}^{n}-\hat{\sigma}_{h k}^{n-1} \|\right|^{2} \\
\quad+\sum_{n=1}^{M} k\left\{\partial \hat{\sigma}_{h k}^{n}, \hat{\tau}^{n}\right\}=\sum_{n=1}^{M} k\left\{\partial \hat{\sigma}_{h k}^{n}, \hat{\tau}^{n}-\hat{\sigma}_{h k}^{n}\right\} .
\end{gathered}
$$

Choosing $\hat{\tau}^{m}=\hat{\sigma}_{h k}^{m}$ for $m<M$, we obtain

$$
\left\{\partial \hat{\sigma}_{h k}^{M}, \hat{\tau}-\hat{\sigma}_{h k}^{M}\right\} \geqq 0, \quad \forall \hat{\tau} \in K_{h}\left(t_{M}\right), \quad M=1, \ldots, N .
$$

It remains to verify that $\hat{\sigma}_{h k}^{n} \in K_{h}\left(t_{n}\right)=\left(E_{h}\left(t_{n}\right) \times V_{h}\right) \cap P$. We recall that $\sigma_{h k \mu}^{n} \in E_{h}\left(t_{n}\right), \sigma_{h k}^{n}=\chi^{n}+\bar{\sigma}^{n}$. Since $E_{h}\left(t_{n}\right)$ is convex and closed in $S$, it is weakly closed in $S$. Furthermore, (2.21) implies that $\sigma_{h k \mu}^{n} \rightarrow \sigma_{h k}^{n}$ weakly in $S$, consequently $\sigma_{h k}^{n} \in E_{h}\left(t_{n}\right)$.

To verify that $\hat{\sigma}_{h k}^{n} \in P$, we use lemma 2.3-(ii). Thus

$$
\begin{gathered}
C \geqq k J_{\mu}\left(\hat{\sigma}_{h k \mu}^{n}\right)=\frac{k}{2 \mu}\left\|\hat{\sigma}_{h k \mu}^{n}-\pi \hat{\sigma}_{h k \mu}^{n}\right\|^{2}, \\
\left\|\hat{\sigma}_{h k}^{n}-\pi \hat{\sigma}_{h k}^{n}\right\|^{2} \leqq \liminf _{\mu \rightarrow 0}\left\|\hat{\sigma}_{h k \mu}^{n}-\pi \hat{\sigma}_{h k}^{n}\right\|^{2} \leqq \liminf _{\mu \rightarrow 0} \frac{2}{k} C \mu=0,
\end{gathered}
$$

which implies $\hat{\sigma}_{h k}^{n} \in P$.

Finally, by virtue of (2.22) and lemma 2.4 , we may write

$$
\sum_{n=1}^{N} k\left\|\partial \hat{\sigma}_{h k}^{n}\right\|^{2} \leqq \sum_{n=1}^{N} k \liminf _{\mu \rightarrow 0}\left\|\partial \hat{\sigma}_{h k}^{n}\right\|^{2} \leqq \liminf _{\mu \rightarrow 0} \sum_{n=1}^{N} k\left\|\partial \hat{\sigma}_{h k \mu}^{n}\right\|^{2} \leqq C,
$$

which concludes the proof of lemma 2.1.

THEOREM 2.1: Let us denote

$$
\varepsilon(h, k)=\inf _{\mathscr{K}}\|\hat{\sigma}-\hat{\tau}\|_{l^{2}(H)}
$$

where

$$
\mathscr{K}=\left\{\hat{\tau}^{n} \in K_{h}\left(t_{n}\right), n=1, \ldots, N\right\}
$$


Assume that if $\Gamma_{\sigma} \neq \emptyset$, there exists

$$
\chi^{0} \in\left[L^{\infty}(\Omega)\right]^{n^{2}} \cap E\left(F^{0}, g^{0}\right)
$$

Then positive constants $C$ and $k_{0}$ exist such that for $k \leqq k_{0}$ :

$$
\max _{n=1, \ldots, N}\left\|\hat{\sigma}^{n}-\hat{\sigma}_{h k}^{n}\right\| \leqq C(\sqrt{\varepsilon}(h, k)+\sqrt{k}) .
$$

Proof: Let us extend $\hat{\sigma}_{h k}^{n}$ onto the whole interval $I$ as follows

$$
\hat{\sigma}_{h k}(t)=\lambda(t) \hat{\sigma}_{h k}^{n-1}+(1-\lambda(t)) \hat{\sigma}_{h k}^{n}, \quad t \in I,
$$

where

$$
\begin{gathered}
\lambda(t)=\frac{\gamma(t)-\gamma\left(t_{n}\right)}{\gamma\left(t_{n-1}\right)-\gamma\left(t_{n}\right)}, \quad t \in I_{n}, \quad \text { if } \gamma\left(t_{n}\right) \neq \gamma\left(t_{n-1}\right), \\
\lambda(t)=\left(t_{n}-t\right) / k, \quad t \in I_{n}, \quad \text { if } \gamma\left(t_{n}\right)=\gamma\left(t_{n-1}\right) .
\end{gathered}
$$

Then for $\hat{\sigma}_{h k}=\left(\sigma_{h k}, \alpha_{h k}\right), \sigma_{h k}(t) \in E(t)$ holds for all $t \in I$. From the convexity of $P$ in $H$ and the fact that $0 \leqq \lambda(t) \leqq 1$ by virtue of (1.4), we obtain $\hat{\sigma}_{h k}(t) \in P$. Consequently, $\hat{\sigma}_{h k}(t) \in K(t)$ and we may insert $\hat{\tau}=\hat{\sigma}_{h k}(t)$ into (1.5) to obtain

$$
\left\{\hat{\sigma}^{\prime}, \hat{\sigma}_{h k}-\hat{\sigma}\right\} \geqq 0 \quad \text { a.e. in } I \text {. }
$$

Integration over $I_{n}$ yields

$$
\left\{\partial \hat{\sigma}^{n}, \hat{\sigma}_{h k}^{n}-\hat{\sigma}^{n}\right\} \geqq \frac{1}{k} \int_{I_{n}}\left\{\hat{\sigma}^{\prime}, \hat{\sigma}_{h k}^{n}-\hat{\sigma}^{n}+\hat{\sigma}(t)-\hat{\sigma}_{h k}(t)\right\} d t .
$$

Let us consider $\hat{\tau}_{h}^{n} \in K_{h}\left(t_{n}\right), n=1, \ldots, N$, such that

$$
\left\|\hat{\sigma}-\hat{\tau}_{h}\right\|_{l^{2}(H)} \leqq 2 \varepsilon(h, k)
$$

and insert $\hat{\tau}=\hat{\tau}_{h}^{n}$ into (2.2). Thus we obtain

$$
\left\{\partial \hat{\sigma}_{h k}^{n}, \hat{\tau}_{h}^{n}-\hat{\sigma}_{h k}^{n}\right\} \geqq 0 \text {. }
$$

For the error $e=\hat{\sigma}-\hat{\sigma}_{h k}$ it follows from (2.24), (2.25):

$$
\left\{\partial e^{n}, e^{n}\right\} \leqq\left\{\partial \hat{\sigma}_{h k}^{n}, \hat{\tau}_{h}^{n}-\hat{\sigma}^{n}\right\}+\left|r_{n}\right|
$$

where $r_{n}$ is the right-hand side of (2.24). Multiplying by $k$ and summing over $n$ yields

$$
\max _{n=1, \ldots, N}\left\|\left|e^{n}\left\|\left.\right|^{2} \leqq C\right\| \partial \hat{\sigma}_{h k}\left\|_{l^{2}(H)}\right\| \hat{\tau}_{h}-\hat{\sigma} \|_{l^{2}(H)}+2 k \sum_{n=1}^{N}\right| r_{n} \mid\right.
$$


For $r_{n}$ we may write

$$
\begin{aligned}
\left|r_{n}\right| \leqq \frac{1}{k} \int_{I_{n}} \| & \hat{\sigma}^{\prime}(t) \|\left[k \mid\left\|\partial \hat{\sigma}_{h k}^{n}\right\|+k^{1 / 2}\left(\left.\int_{I_{n}}\left\|\hat{\sigma}^{\prime}\right\|\right|^{2} d s\right)^{1 / 2}\right] d t \\
& \leqq k^{1 / 2}\left\|\partial \hat{\sigma}_{h k}^{n}\right\|\left\|\left(\int_{I_{n}}\left\|\hat{\sigma}^{\prime}(t)\right\|^{2} d t\right)^{1 / 2}+\int_{I_{n}}\right\| \hat{\sigma}^{\prime}(t)\|\|^{2} d t \\
& \leqq\left. k\left\|\partial \hat{\sigma}_{h k}^{n}\right\|\right|^{2}+\frac{3}{2} \int_{I_{n}}\left\|\hat{\sigma}^{\prime}(t)\right\|^{2} d t .
\end{aligned}
$$

Inserting into (2.27) leads to the following estimate

$$
\begin{aligned}
& \max _{n}\left\|e^{n}\right\|^{2} \leqq 2 C\left\|\partial \hat{\sigma}_{h k}\right\|_{h^{2}(H)} \varepsilon(h, k) \\
&+3 k\left(\sum_{n=1}^{N} k\left\|\partial \hat{\sigma}_{h k}^{n}\right\|\left\|^{2}+\int_{l}\right\| \hat{\sigma}^{\prime}(t) \|^{2} d t\right) \leqq C_{1}(\varepsilon(h, k)+k),
\end{aligned}
$$

if we realize that

$$
\int_{I}\left\|\hat{\sigma}^{\prime}(t) \mid\right\|^{2} d t \leqq C \int_{I}\left(\left\|\sigma^{\prime}(t)\right\|_{S}^{2}+\left\|\alpha^{\prime}\right\|_{L^{2}}^{2}\right) d t<\infty
$$

and use lemma 2.1.

Q.E.D.

\section{A PRIORI ERROR ESTIMATE IN TWO-DIMENSIONAL PROBLEMS}

In the following we shall consider the problems in $R^{2}$ and evaluate the quantity $\varepsilon(h, k)$, introduced in theorem 2.1 , for a piecewise linear finite element model, assuming certain regularity of the exact solution $\hat{\sigma}$.

We assume that the reference body forces $F^{0}$ are constant and the reference surface tractions $g^{0}$ are piecewise linear on $\Gamma_{\boldsymbol{c}}$. reference surface tractions $g^{0}$ are piecewise linear on $\Gamma_{\sigma}$.

Let us consider a regular family $\left\{\mathscr{T}_{h}\right\}, 0<h \leqq h_{0}$, of triangulations of the domain $\Omega$ (i.e., a positive $\vartheta_{0}$ exists such that all angles in all triangulations are not less than $\vartheta_{0}$ ). Let $h$ denote the maximal length of all sides in $\mathscr{T}_{h}$.

We employ the self-equilibriated triangular block-elements of Watwood and Hartz [15], which correspond to the Clough-Tocher element via the slab analogy. The model consists of triangular block-elements, each of them being generated by connecting the vertices of the triangle $K$ with its centre of gravity. On each subtriangle $K_{i}$ three linear functions-components of a selfequilibriated stress tensor - are defined. The stress vector has to be continuous when crossing any common boundary between the subtriangles.

vol. $14, n^{\circ} 4,1980$ 
Some approximability properties of the spaces $N_{h}(\Omega)$, generated by these elements have been studied in [7] and [11]. We shall need the following result (see [7], Thm. 2.3):

Let $\tau \in S \cap\left[C^{2}(\bar{\Omega})\right]^{4}$. Then a linear mapping

$$
r_{h}: \quad E(0,0) \cap\left[C^{2}(\bar{\Omega})\right]^{4} \rightarrow N_{h}(\Omega)
$$

exists such that for any triangle $K \in \mathscr{T}_{h}$ :

$$
\max _{i=1,2,3}\left\|\tau-\left(r_{h} \tau\right)^{i}\right\|_{\left[C\left(K_{i}\right)\right]^{4}} \leqq C h_{K}^{2}\|\tau\|_{\left[C^{2}(K)\right]^{4}}
$$

holds, where $\left(r_{h} \tau\right)^{i}=\left.\left(r_{h} \tau\right)\right|_{K_{1}}, h_{K}$ is the maximal side of $K$ and $C$ is independent of $h_{K}$ and $\tau$.

Let us define the finite element spaces

$$
\begin{gathered}
E_{h}^{0}=N_{h}(\Omega) \cap E(0,0)=\left\{\tau \in N_{h}(\Omega) \mid \tau . \nu=0 \text { on } \Gamma_{\sigma}\right\}, \\
V_{h}=\left\{\beta \in L^{2}(\Omega)|\beta|_{K_{1}} \in P_{1}\left(K_{i}\right), \forall K_{i} \subset K \in \mathscr{T}_{h}\right\} .
\end{gathered}
$$

Note that under the assumptions on $F^{0}$ and $g^{0}$, the auxiliary function $\chi^{0}$ can be chosen piecewise linear with respect to the triangulation $\mathscr{T}_{h_{0}}-$ see remark 2.1. Then $\chi\left(t_{n}\right)=\gamma\left(t_{n}\right) \chi^{0}$ is piecewise linear, as well. In the following, we assume that each $\mathscr{I}_{h}$ of the family $\left\{\mathscr{I}_{h}\right\}$ of the triangulations is generated by a regular refinement of $\mathscr{I}_{h_{0}}$.

THEOREM 3. 1: Let the soiution $\hat{\sigma} \equiv(\sigma, \alpha)$ be such that for $\sigma_{0}=\sigma-\chi$ and $\alpha$ and for any $K^{0} \in \mathscr{T}_{h_{0}}$ :

$$
\begin{gathered}
\sup _{t \in I}\left\|\sigma_{0}(t)\right\|_{\left[C^{2}\left(K^{0}\right]^{4}\right.} \equiv\left\|\sigma_{0}\right\|_{L^{2}\left(I,\left[C^{2}\left(K^{1}\right) I^{+1}\right)\right.}<\infty, \\
\sup _{t \in I}\|\alpha(t)\|_{H^{2}\left(K_{i}^{0}\right)} \equiv\|\alpha\|_{L^{4}\left(I, H^{2}\left(K_{t}^{0}\right)\right)}<\infty, \quad i=1,2,3 .
\end{gathered}
$$

Then

$$
\varepsilon(h, k) \equiv \inf _{\hat{\tau} \in \mathscr{H}}\|\bar{\sigma}-\bar{\tau}\|_{l^{2}(H)} \leqq C h^{2}
$$

holds, where $C=C\left(\sigma_{0}, x\right)$ is independent of $h, k$.

Proof: Let us recall that $\hat{\tau}^{n} \equiv\left(\tau^{n}, \beta^{n}\right), \tau^{n} \in E_{h}\left(t_{n}\right)=\chi^{n}+E_{h}^{0}, \sigma^{n}=\chi^{n}+\sigma_{0}^{n}$, $\tau^{n}=\chi^{n}+\tau_{0}^{n}$, where $\sigma_{0}^{n} \in E(0,0)$ and $\tau_{0}^{n} \in E_{h}^{0}$. Consequently, omitting the superscript $n$, we may write for any $n=1, \ldots, N$ :

$$
\|\hat{\sigma}-\hat{\tau}\|^{2}=\left\|\sigma_{0}-\tau_{0}\right\|_{S}^{2}+\|\alpha-\beta\|_{0, \Omega}^{2} .
$$

Let us set $\tau_{0}=r_{h} \sigma_{0}$. Then $r_{h} \sigma_{0} \in E_{h}^{0}$, because $r_{h} \sigma_{0} . v=0$ follows from the definition of the mapping $r_{h}$ (see [7]). 
Let us consider an arbitrary triangle $K_{i} \subset K, K \in \mathscr{T}_{h}$ and denote its vertices by $a_{j}$. Then if $\beta \in P_{1}\left(K_{i}\right)$ and

$$
\beta\left(a_{j}\right) \geqq f\left(\chi+r_{h} \sigma_{0}\right)\left(a_{j}\right) \equiv d_{j}, \quad j=1,2,3
$$

then

$$
\beta \geqq f\left(\chi+r_{h} \sigma_{0}\right) \text { in } K_{i} .
$$

This follows easily from the linearity of $\chi+r_{h} \sigma_{0}, \beta$ and from the convexity of $f$.

Let $\Pi \alpha \in P_{1}\left(K_{i}\right)$ be the linear Lagrange interpolate of $\alpha$ on $K_{i}$. Define $r \in P_{1}\left(K_{i}\right)$ by the relations

$$
\begin{gathered}
r\left(a_{j}\right)=\max \left(0, d_{j}-\alpha\left(a_{j}\right)\right)=\left[d_{j}-\alpha\left(a_{j}\right)\right]^{+}, \\
\beta_{h}=\Pi \alpha+r \in P_{1}\left(K_{i}\right) .
\end{gathered}
$$

Then obviously $\beta_{h}\left(a_{j}\right) \geqq d_{j}, j=1,2,3$.

It is well-known that

$$
\|\alpha-\Pi \alpha\|_{0, K_{i}} \leqq C h^{2}|\alpha|_{H^{2}\left(K_{i}\right)},
$$

where the seminorm of the second order derivatives stands on the right-hand side $(c f .[2])$.

Using the assumptions (1.1), (1.2) and (3.1), we may write

$$
\begin{aligned}
\left|d_{j}-f\left(\chi+\sigma_{0}\right)\left(a_{j}\right)\right| \leqq \| f(\chi & \left.+r_{h} \sigma_{0}\right)-f\left(\chi+\sigma_{0}\right) \|_{C\left(K_{i}\right)} \\
& \leqq C\left\|\sigma_{0}-r_{h} \sigma_{0}\right\|_{\left[C\left(K_{1}\right]^{4}\right.} \leqq C h^{2}\left\|\sigma_{0}\right\|_{\left[C^{2}\left(K^{0}\right)^{4}\right.} \equiv \varepsilon_{1}(h) .
\end{aligned}
$$

Consequently, we have

$$
\|r\|_{0, K_{1}} \leqq C h \varepsilon_{1}(h) .
$$

From (3.6) and (3.7) we obtain

$$
\text { - }\left\|\alpha-\beta_{h}\right\|_{\theta, K_{1}} \leqq\|\alpha-\Pi \alpha\|_{0, K_{i}}+\|r\|_{0,} \leqq C\left(h^{2}|\alpha|_{H^{2}\left(K_{1}\right)}+h^{3}\left\|\sigma_{0}\right\|_{\left\{C^{2}\left(K^{0}\right)^{4}\right.}\right) \text {. }
$$

Summations of the squares of these estimates leads to the following inequality

$$
\left\|\alpha-\beta_{h}\right\|_{0, \Omega}^{2} \leqq C h^{4}\left(\sum_{K^{0} \in \mathscr{F}_{h_{0}}}\left(\sum_{i=1}^{3}|\alpha|_{H^{2}\left(K_{i}^{0}\right)}^{2}+\left\|\sigma_{0}\right\|_{\left[C^{2}\left(K^{0}\right)\right]^{4}}^{2}\right)\right) .
$$

From (3.1) we conclude that

$$
\left\|\sigma_{0}-r_{h} \sigma_{0}\right\|_{S}^{2} \leqq C h^{4} \sum_{K^{0} \in \mathscr{T}_{h_{0}}}\left\|\sigma_{0}\right\|_{\left[C^{2}\left(K^{0}\right)\right]^{4}}^{2}
$$

vol. $14, \mathrm{n}^{\circ} 4,1980$ 
Inserting (3.8) and (3.9) in (3.2), we obtain

$$
\begin{aligned}
\left\|\hat{\sigma}^{n}-\hat{\tau}^{n}\right\|^{2} \leqq & C h^{4} \sum_{K^{0} \in \mathscr{T}_{h_{0}}}\left(\left\|\sigma_{0}^{n}\right\|_{\left[C^{2}\left(K^{0}\right)\right]^{4}}^{2}+\sum_{i=1}^{3}\left|\alpha^{n}\right|_{H^{2}\left(K_{i}^{0}\right)}^{2}\right) \\
& \leqq C h^{4} \sum_{K^{0} \in \mathscr{T}_{h_{0}}}\left(\left\|\sigma_{0}\right\|_{L^{\times}\left(l,\left[C^{2}\left(K^{0}\right\}^{4}\right)\right.}+\sum_{i=1}^{3}\|\alpha\|_{\left.L^{x}\left(l, H^{2}\left(K_{i}^{0}\right)\right)\right)}^{2}\right)=h^{4} C_{1}\left(\sigma_{0}, \alpha\right) .
\end{aligned}
$$

It is readily seen that

$$
\|\hat{\sigma}-\hat{\tau}\|_{l^{2}(H)}^{2}=\sum_{n=1}^{N} k\left\|\hat{\sigma}^{n}-\hat{\tau}^{n}\right\|^{2} \leqq C_{1}\left(\sigma_{0}, \alpha\right) T h^{4}
$$

and the assertion of the theorem follows.

Q.E.D.

REMARK 3.1: In three-dimensional problems one can employ an analogue of the triangular block-element, i.e. a tetrahedron composed of four subtetrahedrons. Estimates parallel to (3.1) and (3.6) hold-see the forthcoming paper [12] and the book [2], respectively. Thus the approach proposed above leads to the assertion of theorem 3.1, as previously.

THEOREM 3.2: Let the assumptions of theorem 3.1 be satisfied. Then constants $C$ and $k_{0}$ exist such that

$$
\max _{n=1, \ldots, N}\left\|\hat{\sigma}^{n}-\hat{\sigma}_{h k}^{n}\right\| \leqq C(h+\sqrt{k})
$$

holds for $k \leqq k_{0}, h \leqq h_{0}$.

Proof is an immediate consequence of the theorems 2.1 and 3.1.

Remark 3.2: An algorithm for solving the approximate problem (2.2).

Defining $E_{h}^{0}$ and $V_{h}$ as previously, we obtain for $\tau_{0}^{n} \in E_{h}^{0}$ :

$$
\left(\chi^{n}+\tau_{0}^{n}, \beta^{n}\right) \in P \quad \Leftrightarrow \quad \beta^{n}\left(a_{j}\right) \geqq f\left(\chi^{n}+\tau_{0}^{n}\right)\left(a_{j}\right)
$$

at all vertices $a_{j} \in K_{i} \subset K$ of all triangles $K \in \mathscr{T}_{h}$.

Thus we have also nonlinear constraints for the parameters of $\beta^{n}$ and $\tau_{0}^{n}$. (In case of the von Mises yield function-see remark 1.1-these constraints are quadratic.)

At each time level we have to minimize the quadratic functional (2.3) with the nonlinear constraints (3.10) and with linear constraints (equations), which guarantee the continuity of the stress vectors across the interelement boundaries. Therefore we have to choose a suitable algorithm of convec programming (e. g. feasible directions, linearization a. o.). 


\section{CONVERGENCE TO A NON-REGULAR SOLUTION}

First we consider the two-dimensional problems. Let us preserve the assumptions on $F^{0}$ and $g^{0}$ from section 3 , so that the functions $\chi^{0}$ and $\chi\left(t_{n}\right)$ are piecewise linear with respect to the triangulation $\mathscr{T}_{h_{0}}$. The main result of the present section is the following.

THEOREM 4.1: Let us assume that:

(i) if $\Gamma=\Gamma_{\sigma}$, then $\Omega$ is a starlike domain;

(ii) if $\Gamma=\bar{\Gamma}_{u} \cup \bar{\Gamma}_{\sigma}$, there exists a point $A \in R^{2}$ such that if $A$ coincides with the origin, then for $\lambda=1+\varepsilon$ and $\varepsilon>0$ sufficiently small, either

$$
\lambda \bar{\Gamma}_{\sigma} \subset R^{2} \dot{-} \Omega \quad \text { or } \quad \lambda \bar{\Gamma}_{\sigma} \subset \Omega .
$$

Here $\lambda \bar{\Gamma}_{\sigma}$ denotes the image of $\bar{\Gamma}_{\sigma}$ by the dilatation mapping $y=\lambda x$.

Let the family $\left\{\mathscr{I}_{h}\right\}$ of triangulations be generated by regular refinements of the triangulation $\mathscr{I}_{h_{0}}$.

Then for any $k$ sufficiently small

$$
\lim _{h \rightarrow 0} \inf _{\hat{\tau} \in \mathcal{X}}\|\hat{\sigma}-\hat{\tau}\|_{I^{2}(H)}=0 .
$$

In the proof we shall need the following:

Proposition 4.1: Let the assumptions (i), (ii) of theorem 4.1 be satisfied. Then the set

$$
E(0,0) \cap\left[C^{\infty}(\bar{\Omega})\right]^{4}
$$

is dense in $E(0,0)$ (with respect to the $L^{2}$-norm).

For the proof, see [7], theorems 4.1-4.3.

REMARK 4.1: A more general result could be proven for the case $\Gamma=\Gamma_{\sigma}$, by means of the Airy stress function [13].

Proof of theorem 4.1: Let us consider a fixed time $t_{n}$ and let us omit the superscript $n$. Using proposition 4. 1, one can find $\sigma_{0 \varepsilon} \in E(0,0) \cap\left[C^{\infty}(\bar{\Omega})\right]^{4}$ such that

$$
\left\|\sigma_{0}-\sigma_{0 \varepsilon}\right\|_{s} \leqq \varepsilon .
$$

By a regularization of $\alpha$, we obtain $\alpha_{\varepsilon} \in C^{\infty}(\bar{\Omega})$ such that

$$
\left\|\alpha-\alpha_{\varepsilon}\right\|_{0, \Omega} \leqq \varepsilon \text {. }
$$

Defining

$$
\mathscr{F} \equiv f\left(\chi+\sigma_{0}\right)-\alpha, \quad \mathscr{F}_{\varepsilon}=f\left(\chi+\sigma_{0 \varepsilon}\right)-\alpha_{\varepsilon},
$$

we see that $\mathscr{F} \leqq 0$ a. e. in $\Omega$, but the same inequality is not true for $\mathscr{F}_{\varepsilon}$, in general. vol. $14, n^{\circ} 4,1980$ 
Note that $\chi \in\left[P_{1}\left(K^{0}\right)\right]^{4}$ for all $K^{0} \in \mathscr{T}_{h_{0}}$. We choose $\hat{\tau}=\left(\chi+\tau_{0}, \beta_{h}\right)$, where $\tau_{0}=r_{h} \sigma_{0 \varepsilon}, \beta_{h}=\Pi_{h} \alpha_{\varepsilon}+\rho$; here $\Pi_{h} \alpha_{\varepsilon}$ and $\rho$ are defined locally in every $K_{i} \subset K \subset K^{0}$, as follows: $\Pi_{h} \alpha_{\varepsilon}$ coincides with the linear Lagrange interpolate $\Pi_{K_{1}} \alpha_{\varepsilon}$ of $\alpha_{\varepsilon}$ on $K_{i}$,

$$
\rho \in P_{1}\left(K_{i}\right), \quad \rho\left(a_{j}\right)=\left(d_{j}-\alpha_{\varepsilon}\left(a_{j}\right)\right)^{+}, \quad j=1,2,3
$$

where $a_{j}$ are the vertices of the subtriangle $K_{i}$ and

$$
d_{j}=f\left(\chi+r_{h} \sigma_{0 \varepsilon}\right)\left(a_{j}\right) .
$$

It is easy to verify that $\chi+r_{h} \sigma_{0 \varepsilon} \in E\left(t_{n}\right)$ and

$$
f\left(\chi+r_{h} \sigma_{0 \varepsilon}\right)-\beta_{h} \leqq 0 \quad \text { a.e. in } \Omega \text {. }
$$

Next we have to estimate $\rho$ in $L^{2}(\Omega)$. We may write for any $j=1,2,3$ :

$$
0 \leqq \rho\left(a_{j}\right)=\left(d_{j}-\alpha_{\varepsilon}\left(a_{j}\right)\right)^{+} \leqq\left|d_{j}-f\left(\chi+\sigma_{0 \varepsilon}\right)\left(a_{j}\right)\right|+\mathscr{F}_{\varepsilon}^{+}\left(a_{j}\right),
$$

since

$$
-\alpha_{\varepsilon}\left(a_{j}\right) \leqq \mathscr{F}_{\varepsilon}^{+}\left(a_{j}\right)-f\left(\chi+\sigma_{0 \varepsilon}\right)\left(a_{j}\right) .
$$

Moreover, we have

$$
\left|d_{j}-f\left(\chi+\sigma_{0 \varepsilon}\right)\left(a_{j}\right)\right| \leqq C h^{2}\left\|\sigma_{0 \varepsilon}\right\|_{\left[C^{2}(\bar{\Omega})\right]^{4}} \equiv \varepsilon_{1}\left(h, \sigma_{0 \varepsilon}\right)
$$

and therefore

$$
\left.\begin{array}{c}
\rho \leqq \varepsilon_{1}\left(h, \sigma_{0 \varepsilon}\right)+\Pi_{K_{i}} \mathscr{F}_{\varepsilon}^{+} \text {on } K_{i} \\
\|\rho\|_{0, K_{t}}^{2} \leqq 2 \varepsilon_{1}^{2} \operatorname{mes} K_{i}+2\left\|\Pi_{K_{i}} \mathscr{F}_{\varepsilon}^{+}\right\|_{0, K_{i}}^{2}
\end{array}\right\}
$$

LEMMA 4.1: The following estimate holds

$$
\left\|\mathscr{F}_{\varepsilon}^{+}\right\|_{0, K^{0}} \leqq C \varepsilon, \quad \forall K^{0} \in \mathscr{T}_{h_{0}} .
$$

Proof: Using (1.1) and (1.2), we derive

$$
\begin{gathered}
\left|f\left(\chi+\sigma_{0}\right)-f\left(\chi+\sigma_{0 \varepsilon}\right)\right| \leqq C\left\|\sigma_{0}-\sigma_{0 \varepsilon}\right\|_{\mathbb{R}_{\sigma^{\prime}}} \\
\left\|f\left(\chi+\sigma_{0}\right)-f\left(\chi+\sigma_{0 \varepsilon}\right)\right\|_{0, K^{0}}^{2} \leqq C\left\|\sigma_{0}-\sigma_{0 \varepsilon}\right\|_{S}^{2} \leqq C \varepsilon^{2}
\end{gathered}
$$

and therefore

$$
\left\|\mathscr{F}-\mathscr{F}_{\varepsilon}\right\|_{0, K^{0}} \leqq\left\|f\left(\chi+\sigma_{0}\right)-f\left(\chi+\sigma_{0 \varepsilon}\right)\right\|_{0, K^{0}}+\left\|\alpha-\alpha_{\varepsilon}\right\|_{0, K^{0}} \leqq C \varepsilon .
$$

Furthermore, denoting $\Omega_{1}=\operatorname{supp} \mathscr{F}_{\varepsilon}^{+} \cap K^{0}$, we have

$$
\left\|\mathscr{F}_{\varepsilon}^{+}\right\|_{0, K^{0}}^{2} \leqq \int_{\Omega_{1}}\left(\mathscr{F}_{\varepsilon}^{+}-\mathscr{F}^{2}\right)^{2} d x=\left\|\mathscr{F}_{\varepsilon}-\mathscr{F}\right\|_{0, \Omega_{1}}^{2} \leqq C \varepsilon^{2} .
$$


LeMma 4. 2: Let $\Pi_{h} \mathscr{F}_{\varepsilon}^{+}$be defined locally on every $K_{i} \subset K \subset K^{0}$ by the linear Lagrange interpolate $\Pi_{K_{1}} \mathscr{F}_{\varepsilon}^{+}$. Then

$$
\left\|\mathscr{F}_{\varepsilon}^{+}-\Pi_{h} \mathscr{F}_{\varepsilon}^{+}\right\|_{0, K^{0}} \leqq \varepsilon_{2}(h), \quad \varepsilon_{2}(h) \rightarrow 0 \text { for } h \rightarrow 0
$$

holds for any $K^{0} \in \mathscr{T}_{h_{0}}$.

Proof: Since $\mathscr{F}_{\varepsilon} \in C\left(K^{0}\right)$, also $\mathscr{F}_{\varepsilon}^{+} \in C\left(K^{0}\right)$. For any $\eta>0$ there exists a polynomial $p$ such that

$$
\left\|\mathscr{F}_{\varepsilon}^{+}-p\right\|_{C\left(K^{0}\right)} \leqq \eta \text {. }
$$

Moreover, we have for any $K_{l} \subset K \subset K^{0}$ :

$$
\begin{gathered}
\left\|\Pi_{h} p-\Pi_{h} \mathscr{F}_{\varepsilon}^{+}\right\|_{C\left(K_{t}\right)} \leqq\left\|p-\mathscr{F}_{\varepsilon}^{+}\right\|_{C\left(K_{i}\right)} \leqq\left\|p-\mathscr{F}_{\varepsilon}^{+}\right\|_{C\left(K^{0}\right)}, \\
\left\|p-\Pi_{h} p\right\|_{C\left(K_{t}\right)} \leqq C_{0} h^{2}\|p\|_{C^{2}\left(K^{0}\right)} .
\end{gathered}
$$

Consequently, we may write

$$
\begin{aligned}
\left\|\mathscr{F}_{\varepsilon}^{+}-\Pi_{h} \mathscr{F}_{\varepsilon}^{+}\right\|_{C\left(K^{0}\right)} \leqq & \left\|\mathscr{F}_{\varepsilon}^{+}-p\right\|_{C\left(K^{0}\right)}+\left\|p-\Pi_{h} p\right\|_{C\left(K^{0}\right)} \\
& +\left\|\Pi_{h} p-\Pi_{h} \mathscr{F}_{\varepsilon}^{+}\right\|_{C\left(K^{0}\right)} \leqq 2 \eta+C_{0} h^{2}\|p\|_{C^{2}\left(K^{0}\right)}=\delta\left(h, K^{0}\right)
\end{aligned}
$$

and (4.4) follows with

$$
\varepsilon_{2}(h)=\max _{K^{0} \in \mathscr{T}_{h_{0}}}\left(\delta\left(h, K^{0}\right) \sqrt{\operatorname{mes}} K^{0}\right) .
$$

Q.E.D.

Using lemmas 4.1, 4.2 and (4.3), we deduce

$$
\begin{aligned}
&\|\rho\|_{0, K^{0}}^{2} \leqq 2 \varepsilon_{1}^{2} \operatorname{mes} K^{0}+4\left(\left\|\mathscr{F}_{\varepsilon}^{+}\right\|_{0, K^{0}}^{2}+\left\|\Pi_{h} \mathscr{F}_{\varepsilon}^{+}-\mathscr{F}_{\varepsilon}^{+}\right\|_{0, K^{0}}^{2}\right) \\
& \leqq 2 \varepsilon_{1}^{2} \operatorname{mes} K^{0}+C\left(\varepsilon^{2}+\varepsilon_{2}^{2}\right) .
\end{aligned}
$$

Altogether, we have

$$
\begin{aligned}
&\|\hat{\sigma}-\hat{\tau}\|^{2}=\| \sigma_{0}- r_{h} \sigma_{0 \varepsilon}\left\|_{S}^{2}+\right\| \alpha-\beta_{h} \|_{0, \Omega}^{2} \leqq 2\left(\left\|\sigma_{0}-\sigma_{0 \varepsilon}\right\|_{S}^{2}\right. \\
&+\| \sigma_{0 \varepsilon}-\left.r_{h} \sigma_{0 \varepsilon} \|_{S}^{2}\right)+3\left(\left\|\alpha-\alpha_{\varepsilon}\right\|_{0, \Omega}^{2}+\left\|\alpha_{\varepsilon}-\Pi_{h} \alpha_{\varepsilon}\right\|_{0, \Omega}^{2}+\|\rho\|_{0, \Omega}^{2}\right) \\
& \leqq 2\left(\varepsilon^{2}+C h^{4}\left\|\sigma_{0 \varepsilon}\right\|_{\left[C^{2}(\bar{\Omega})\right]^{4}}^{2}\right)+C\left(\varepsilon^{2}+h^{4}\left|\alpha_{\varepsilon}\right|_{H^{2}(\Omega)}^{2}\right. \\
&\left.\quad+h^{4}\left\|\sigma_{0 \varepsilon}\right\|_{\left\{C^{2}(\bar{\Omega})\right\}^{4}}^{2}+\varepsilon^{2}+\varepsilon_{2}^{2}(h)\right) .
\end{aligned}
$$

Consequently,

$$
\lim _{h \rightarrow 0}\left\|\hat{\sigma}^{n}-\hat{\tau}\right\|=0, \quad n=1, \ldots, N
$$

If $k=T / N$ is fixed, we are led to the assertion of theorem 4.1.

REMARK 4.2: In three-dimensional problems, the theorem 4.1 can be proved, if we use tetrahedral block-elements (see remark 3.1). As the density of infinitely smooth functions in $E(0,0)$ is concerned, an analogue of proposition 4.1 in $R^{3}$ follows by means of the approach used in [7]. 


\section{REFERENCES}

1. I. BabuŠka, M. Práger and E. Vitásek, Numerical Processes in Differential Equations, S.N.T.L., Prague, 1966.

2. P. G. Ciarlet, The Finite Element Method for Elliptic Problems, North Holland Publ. Comp., Amsterdam, 1978.

3. K. Gróger, Initial Value Problems for Elastoplastic and Elasto-Viscoplastic Systems. Nonlinear Analysis, Proc. Spring School, Teubner, Leipzig, 1979, pp. 95-127.

4. B. Halphen and Nguyen Quoc Son, Sur les matériaux standard généralisés, J. Mécan., Vol. 14, 1975, pp. 39-63.

5. R. Hill, Mathematical Theory of Plasticity, Oxford, 1950.

6. I. Hlaváčex and J. NeČAs, Mathematical Theory of Elastic and Elasto-Plastic Bodies, Elsevier, Amsterdam, 1980.

7. I. Hlaváčer, Convergence of an Equilibrium Finite Element Model for Plane Elastostatics, Apl. Mat., Vol. 24, 1979, pp. 427-457.

8. C. Johnson, On Plasticity with Hardening, J. Math. Anal. Appl., Vol. 62, 1978, pp. 325-336.

9. C. Johnson, A Mixed Finite Element Method for Plasticity Problems with Hardening, S.I.A.M. J. Numer. Anal., Vol. 14, 1977, pp. 575-583.

10. C. Johnson, On Finite Element Methods for Plasticity Problems, Numer. Math., Vol. 26, 1976, pp. 79-84.

11. C. Johnson and B. Mercier, Some Equilibrium Finite Element Methods for TwoDimensional Elasticity Problems, Numer. Math., Vol. 30, 1978, pp. 103-116.

12. M. K ŘižzK, An Equilibrium Finite Element Method in Three-Dimensional Elasticity, Apl. Mat. (to appear).

13. B. Mercier, A personal communication.

14. NGuyen Quoc Son, Matériaux élastoplastiques écrouissabie, Arch. Míech. Stos., Vol. 25, 1973, pp. 695-702.

15. V.B. Watwood and B. J. HaRTZ, An Equilibrium Stress Field Model for Finite Element Solution of Two-Dimensional Elastostatic Problems, Inter. J. Solids Structures, Vol. 4, 1968, pp. 857-873. 